\title{
Research on PID Neural Network Control System of Temperature for Agricutural Film Unit
}

\author{
Feng Yue, Qiao Meixia, Zheng Shuai \\ College of Automation \\ Harbin University of Science and Technology \\ Harbin, Heilongjiang Province, China \\ E-mail:qiaomeixiayx@sina.cn
}

\begin{abstract}
The temperature of agricultural film unit affects the plastic film directly. Since unit heating process has the characters of time delay, nonlinear, time-varying and strong coupling. It is difficult to create a mathematical model structure of plastic melting process. Thus, temperature control is very difficult. This paper presents decoupling control strategy and corresponding control algorithm based on PID (proportional- Integral- differential) neural network. Proportional, integral, differential neurons form a three-layer neural network. This design gives full play to respective advantages of PID control and neural network, and takes advantage of BP neural network to establish the dynamic model of system.
\end{abstract}

\section{Keywords-PID ANN; Agricultural film; Dynamic model}

\section{INTRODUCTION}

The agricultural film unit makes agricultural film in onetime. It is now widely used in various plastic processing industries. Raw material temperature in the channels of blown film machine will directly impact on the quality of agricultural films. Therefore, temperature control accuracy of agricultural film unit is the key to achieve precision molding.

The agricultural film unit heating process is a complex industrial process. And unit heating process has the characters of time delay, nonlinear, time-varying and strong coupling. It is difficult to create a mathematical model structure of plastic melting process. PID is widely used in the control strategy [1].The parameters of PID need to be adjusted regularly. Tuning PID parameters mainly depends on the experience of engineers, and takes much time. But it is too hard for general operating personnel. With the development of control theory and technology, to control the delay complicated object, some scholars have proposed Smith predictor, predictive control methods and so on [2-3], which is established by the basis of the process model. If model mismatches, it will lead to poor quality. In addition, S. N. Huang with others proposed adaptive generalized predictive control of barrel temperature [4]. It is very effective by this method, but online calculation is large. C. $\mathrm{H}$. $\mathrm{Lu}$ with others proposed multivariable self-tuning predictive control [5] and adaptive decoupling control strategy [6]. It is effective in control accuracy. But by utilizing off-line identification method, the robustness of control system is poor.
In view of this, this paper presents a kind of PID neural network decoupling control strategy for agricultural film unit heating process. Through simulation, the control strategy has good adaptive capacity. It improves the dynamic characteristics of system, and solves the problems of material temperature and material supply rate coupled control.

\section{PROCESS PRINCIPLE ANALYSIS}

The agricultural film unit completes agricultural film process. This process generally includes pre-molding, extrusion, measurement, blown stereotypes and so on. Preplasticization metering process makes granular plastic into homogenizer mucus state. Mucus is extruded into blown film die cavity by extrusion cylinder. A certain pressure of air is used to blown film and stretch stereotypes. Then products formed. Agricultural film unit is constituted by extruder barrel and die head machine body. The one end of extruder barrel is a hopper, The other end is connected to the die head. The barrel is generally divided into several thermostatic heating intervals. Each of the heating intervals has a separate heater. Induction heater inverter is used, as shown in Figure 1. The temperature setting value of each heating intervals has a stepped distribution along the extruder barrel. Plastic absorbs heat and gradually reaches molten state. Plastic goes into die head under screw driven. In order to ensure melt quality, it is necessary to set reasonable intervals of extrusion barrel temperature and control temperature strictly.

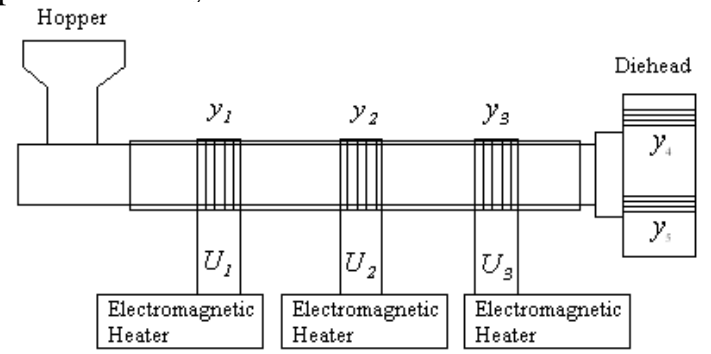

Figure 1. The agricultural film unit heating process model

As using electromagnetic induction heating, the heat is concentrated at the inside surface of the sensing area of the agricultural film unit. The cylinder wall has a certain thickness. Thermocouple is placed in a certain depth of the agricultural film unit cylinder. Agricultural film unit mechanical body is used to be conducted detected 
temperature. The cooling system uses water to cool the outer surface of the agricultural film unit. Therefore, the plastic temperature rising and cooling process is a typical large time delay, nonlinear process. In addition, with the temperature increasing, the specific heat capacity of plastic, the parameters of thermal diffusion and thermal system will be changed. The temperature control object is a time-varying complex process. It is too difficult to establish the mathematical model.

At the same time, the control of material constant temperature and material supply rate of extrusion links is obvious coupling.

\section{BASED ON PID NEURAL NETWOEK DECOUPLING CONTROL STRATEGY}

Traditional control methods rely on the mathematical model of control object. The mathematical model of an object must be known and linear time-invariant. In the agricultural film unit heating systems, it is very difficult to find mathematical model between material temperature and material supply speed, which must have be known and linear time-invariant. Even if the conditions are met, system decoupling requirements and control performance are difficult to be balanced. In addition, the changing of thermal diffusion loss makes control more complex. All of these aspects result in the failure of traditional decoupling algorithm, which depends on the object parameters and object model.

\section{A. The Structure of PID Neural Network Controller}

Agricultural film unit controller which is based on PID neural network is consisted of Proportion (P), integral (I), Derivative (D) neurons. The number, connection, connection weights initial value of neurons in layers are determined by the basic principles of PID control law. It has the advantages of PID control and neural network. Its structure is shown in Figure 2. In the Figure, T0 and f0 represent system setting value. These are the set value of materials temperature and material supply speed. And v1 and v2 represent the output value of PID neural network, used to control the corresponding induction heating power supply power and supplied to the motor. $\mathrm{T}$ and $\mathrm{f}$ represent the actual value of system, that is actual material temperature and supply motor speed. PIDNN neural network through online learning, it adjusts connection weights, changes the strength of network proportional, integral and derivative, based on the changing between object parameters and performance of system output. So system will be in good dynamic and static and achieve the purpose of decoupling control.

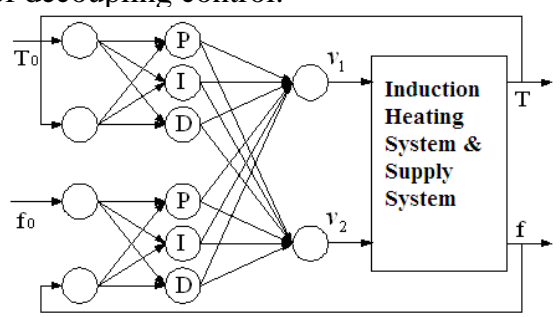

Figure 2. The structure of PID neuron network decoupling control system

\section{B. Control Algorithm}

PID neural network calculation includes forward algorithm and back propagation algorithm.

\section{1) Forward algorithm}

For any sampling instant $k$, forward PID neural network is calculated as follows.

Input and output function of input layer neurons:

$$
x_{s i}(k)=u_{s i}(k)
$$

In the formula: $u_{s i}$ is input value of input layer neurons, $x_{s i}$ is output value of input layer neurons, $s$ is the serial number of the subnet tied, $s=1,2 ; i=1,2$.

The imports value of hidden layer unit has the same formula, that is:

$$
u_{s j}^{\prime}(k)=\sum_{i=1}^{2} w_{s i j} x_{s i}(k)
$$

There are three kinds of state function in the hidden layer neurons. When the output limits, the proportional neuron output is:

$$
x_{s 1}^{\prime}(k)=u_{s 1}^{\prime}(k)
$$

The output of Integral element is:

$$
x_{s 2}{ }^{\prime}(k)=x_{s 2}{ }^{\prime}(k-1)+u_{s 2}{ }^{\prime}(k)
$$

The output of the differential element:

$$
x_{s 3}{ }^{\prime}(k)=u_{s 3}{ }^{\prime}(k)-u_{s 3}{ }^{\prime}(k)
$$

In the formula from (2) to (5): $u_{s j}{ }^{\prime}$ is hidden layer neuron input value, $x_{s j}{ }^{\prime}$ is hidden layer neurons output value, $w_{s i j}$ is each of subnets input layer with hidden layer connection weights, $j=1,2,3 ; s=1,2$.

The input of output layer neurons is weighted in sum of the hidden layer neurons output value:

$$
u_{h}^{\prime \prime}(k)=\sum_{s=1}^{2} \sum_{j=1}^{3} w_{s j h}^{\prime} x_{s j}^{\prime}(k)
$$

The output of output neurons:

$$
x_{h}^{\prime \prime}(k)=u_{h}^{\prime \prime}(k)
$$

In the formula from (6) to (7): $u_{h}^{\prime \prime}$ is output layer neuron input values, $x_{h}^{\prime \prime}$ is output layer neuron output value, $w_{s j h}^{\prime}$ is 
connection weights of hidden layer with output layer, $h=1,2$ is output layer neuron number.

\section{2) Back Propagation Algorithm}

PID neural network learning goal is to make

$$
J=\sum_{p=1}^{2} E_{p}=\frac{1}{m} \sum_{p=1}^{2} \sum_{k=1}^{m}\left[r_{p}(k)-y_{p}(k)\right]^{2}=\frac{1}{m} \sum_{p=1}^{2} \sum_{k=1}^{m} e_{p}^{2}(k)
$$

minimum. In the formula, $m$ is sampling points of each batch, $p$ is the number of system variables. It uses gradient method to adjust the PID neural network weights. Based on $n_{0}$ step training and learning weights were determined by the following formulas.

Hidden layer to the output layer weights iterative formula is below.

$$
\begin{gathered}
w_{s j h}^{\prime}\left(n_{0}+1\right)=w_{s j h}^{\prime}\left(n_{0}\right)-\eta_{s j h}^{\prime} \frac{\partial J}{\partial w_{s j h}^{\prime}} \\
\frac{\partial J}{\partial w_{s j h}^{\prime}}=-\frac{1}{m} \sum_{p=1}^{2} \sum_{k=1}^{m}\left[\delta_{h p}^{\prime}(k) x_{s j}^{\prime}(k)\right] \\
\delta_{h p}^{\prime}(k)=-\frac{2}{m}\left[r_{p}(k)-y_{p}(k)\right] \operatorname{sgn} \frac{y_{p}(k+1)-y_{p}(k)}{v_{h}(k)-v_{h}(k-1)}
\end{gathered}
$$

Input layer to the hidden layer weights iterative formula is below.

$$
\begin{gathered}
w_{s i j}{ }^{\prime}\left(n_{0}+1\right)=w_{s i j}\left(n_{0}\right)-\eta_{s i j} \frac{\partial J}{\partial w_{s i j}} \\
\frac{\partial J}{\partial w_{s i j}}=-\frac{1}{m} \sum_{p=1}^{2} \sum_{h=1}^{2} \sum_{k=1}^{m} \delta_{s j h}(k) x_{s i}(k) \\
\delta_{s j h}(k)=\delta_{h p}^{\prime}(k) w_{s j h}^{\prime} \operatorname{sgn} \frac{x_{s j}^{\prime}(k)-x_{s j}^{\prime}(k-1)}{u_{s j}^{\prime}(k)-u_{s j}^{\prime}(k-1)}
\end{gathered}
$$

For structures and algorithms of control system, PID neural network controller is not dependent on recognition of controlled object mathematical model, but control effect online self-learn and network connection weight values.

\section{The Establishment OF System Model}

In this paper, used offline training methods, it establishes dynamic model of system structure which is shown in Figure 3. Multilayer feedforward neural networks can let any precision approach any nonlinear mapping. It uses BP neural network to create a dynamic model between material temperature and material supply speed. Through direct learning system input / output data, the error function $e(k)$ between actual system output value and BP neural output is less than the target set. The relationship between input and output data is summed which is implicated in the system. $H(k)$ can approximate the same input signal inspired objects output $Y(k)$.The identification of original system and the establishment of system dynamics model are completed.

System model selects three-layer forward neural network. The number of neurons layers is: four input layers, six hidden layers and two output layers. The special function is Sigmoid. Model order is one. A function $e(k)$ between the system actual output and the BP neural network output adjusts and trains the neural network. Trained BP neural network shall be the required dynamic model.

PID neural network decoupling control system can make BP neural network to do objects offline training and get PID neural network weights. Each batch of sample points $m$ is 200, controlled variable $n$ is 2 . The learning step $\eta$ is 0.01 .

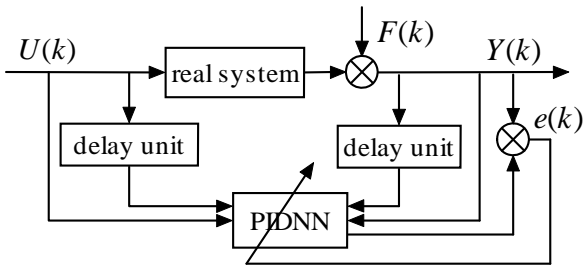

Figure 3. Diagram of dynamic model structure

\section{EXPERIMENTAL RESULTS AND ANALYSIS}

Based on PID neural network decoupling control algorithm, the agricultural film unit heating system input is step signal control simulation. The material temperature is setted to $200{ }^{\circ} \mathrm{C}$. Compared with the traditional PID control strategy, simulation response is shown in Figure 4.

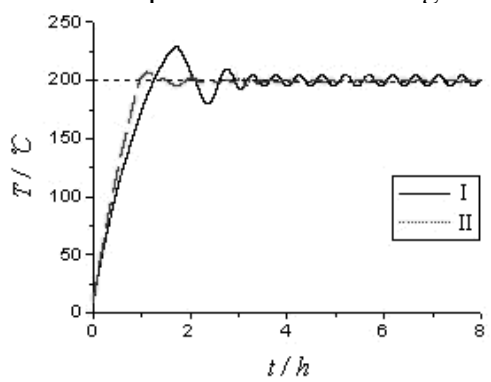

I . PID control; II . PID Neural Network control;

Figure 4. System response comparison between PID-NN and PID control

The simulation results show that PID neural network control strategy compared to PID control strategy can improve the dynamic characteristics of the controlled object, effectively inhibit stable periodic oscillation phenomenon, and make significant reduction in response time and 
overshoot. Overshoot control is within $\pm 7^{\circ} \mathrm{C}$. The steadystate accuracy is $\pm 2^{\circ} \mathrm{C}$. Thinking of the actual production, there is a certain disturbance factors. To further verify the robustness of PID neural networks control method and traditional neural network, the system enters steady state, a short cycle sudden disturbance is plus, the system response curve is shown in Figure 5 and Figure 6.

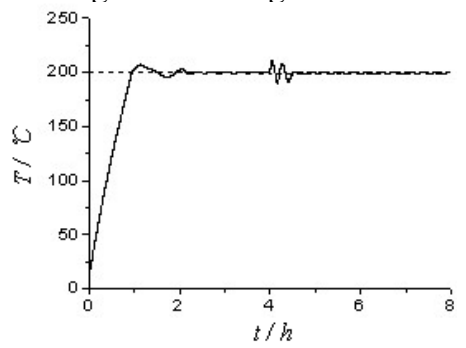

Figure 5. Traditional neural network control disturbance response

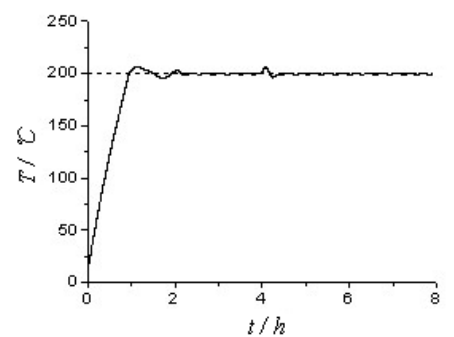

Figure 6. PID neural network to control the disturbance response

Comparative analysis of Figures 5 and 6, disturbance is plus the traditional neural network control strategy. When entering disturbance, the control system can be gradually stabilized. But there has been a large oscillation. For PID neural network control strategy, the system goes into stable state quickly. The system response has a certain overshoot. But the control performance has been improved. It is controllability.

To change materials supplied speed setting (motor frequency) is to measure induction heating system output power (thermostatically controlled) and supplied speed to verify trained the effect of PID neural network decoupling control system. Actual measurement result is shown in Figure 7. In the 50s, the motor frequency settings are changed from $30 \mathrm{~Hz}$ to $50 \mathrm{~Hz}$. PID neural network controls the induction heating system power value based on the new material supply rate. Heating system power value is $14 \mathrm{KW}$. The material temperature is no change and within the range of $200^{\circ} \mathrm{C}$. The quality of the production of the agricultural film is ensured. So PID neural network has a good decoupling control effect.

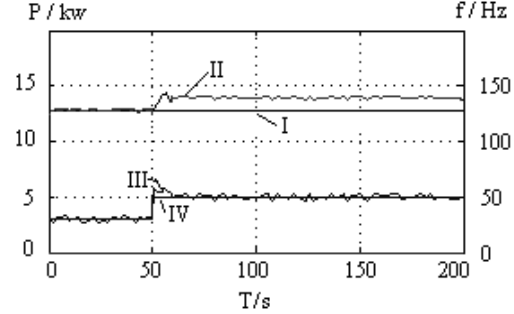

I Actual heating power; II Setting heating power;

III Seting supply motor speed; IV actual setting supply motor speed

Figure 7. The relationship between heating power supplied and motor speed in PIDNN decoupling control

\section{CONCLUSION}

According to agricultural film unit heating production process temperature is difficulty to control. This paper presents a PID neural network decoupling control strategy, and uses BP neural network to establish the dynamic model of system. Through simulation, the control strategy can improve the system itself and system dynamic performance and robustness. As an intelligent control algorithm, it can solve the coupling problem between temperature control of heating power and material feeding speed in the agricultural film production.

\section{ACKNOWLEDGMENT}

This project was sponsored by Heilongjiang Province Science and Technology Research Funds (Project Number GZ11A204).

\section{REFERENCES}

[1] AHANG Qi-miao, AHANG Pei-ren, and WANG Kang-zheng, "Design of Temperature Control System of Plastic Injecting Machine,” J. Automation \& Instrumentation, vol. 2, 2005, pp. 29-31.

[2] Wang S H, Xu B G, and Wang Q Y, et al, "Modified Smith predictor and controller for time-delay process with uncertainty," C. Dalian, China: The $6^{\text {th }}$ World Congress on Intelligent Control and Automation, vol. 1, June, 2006, pp. 623-627.

[3] Jiang Tiezheng, Chen Chen, and Ai Qian, "Nonlinear optimal predictive controller for main steam valve of turbine generator," J. Control Theory and Application, vol. 23(3), March, 2006, pp. 358462.

[4] S. N.Huang, K. K. Tan, and T. H. Lee, "Adaptive GPC control of melt temperature in injection moulding,” J. ISA Transaction, vol. 38, 1999, pp. 361-373.

[5] CC Tsai, C. H. Lu, "Multivariable Self-Tuning Temperature Control for Plastic Injection Molding Process,” J. Industry Application, vol. 34(2), March/April, 1998, pp. 310-318.

[6] C. H. Lu, CC Tsai, "Adptive Decoupling Predictive Temperature Control for an Ectrusion Barrel in a Plastic Injection Molding Process,” J. IEEE Transations on Industry Electronics, vol. 48(5), 2001, pp. 263-268. 